\title{
Asymptotic estimates for finite-time ruin probability of a bidimensional risk model based on entrance process
}

\section{Zhankui Wang}

\begin{abstract}
Consider a bidimensional risk model based on entrance process with constant force of interest in which the claim size from the same business are heavy-tailed and pairwise strong quasi-asymptotically independent, the two counting processes of different business satisfy a certain dependence structure. A precise asymptotic formula for the finite-time ruin probability is obtained.
\end{abstract}

Index Terms - Bidimensional risk model; ruin probability; independence; asymptotic formula $\operatorname{MSC}(2010):-60 \mathrm{~B} 12$, $91 B 30$

\section{INTRODUCTION}

We know that the literature [1] put forward into a new model (LIG model) based on an entrance process and discussed asymptotic normality of the risk process. Furthermore, some scholars got some conclusions through the study of the LIG model. [2-4] investigated the one-dimensional risk model based on entrance processes. Recently, more attention has been paid to multi-dimensional risk models, especially bidimensional ones. [5] discussed the precise large deviations based on the entry process risk model in the independent case of multi-risk. [6] studied the ruin probability of a bidimensional risk model based on entrance processes with constant interest rate.

In this paper, we investigate finite-time ruin probability of a bidimensional risk model based on entrance processes, in which an insurance company operates two kinds of business. Suppose that the initial insurance fund for the $i$-th class is $x_{i}$ and $S_{j}^{i}$ is entry time of the $j-t h$ policy with $0<S_{1}^{i}<S_{2}^{i}<\cdots$ and $S_{j}^{i}=\sum_{k=1}^{j} \theta_{k}^{i}$, $i=1,2 .\left\{\left(N_{1}(t), N_{2}(t)\right)^{\mathrm{T}}\right.$,

$t \geq 0\}$ is a bidimensional renewal counting process. Here, $N_{i}(t)=\sup \left\{j \geq 0: S_{j}^{i} \leq t\right\}, t \geq 0, i=1,2$. For more detail of a bidimensional renewal counting process, we refer the reader to Examples 3.1 and 3.2 of [7]. Denote the mean function by $\lambda_{i}(t)=E N_{k}(t)$ with $\lambda_{i}(0)=0$ and $\lambda_{i}(t)<\infty$, and define the set $\Lambda_{i}=\left\{t>0: \lambda_{i}(t)>0\right\}=\left\{t>0: P\left(S_{1}^{i} \leq t\right)\right\}$. If we

Zhankui Wang, School of Mathematics and Statistics, Northwest Normal University, Lanzhou, Gansu, China, Mobile No. 18894575336 set $t_{-}^{i}=\inf \left\{t>0: P\left(S_{1}^{i} \leq t\right)>0\right\}$, then it is easy to see that $\Lambda_{i}=\left[t_{-}^{i}, \infty\right]$ if $P\left(S_{1}^{i}=t_{-}^{i}\right)>0$; or $\Lambda_{i}=\left(t_{-}^{i}, \infty\right]$ if $P\left(S_{1}^{i}=t_{-}^{i}\right)=0, i=1,2$. We denote the intersection set by $\Lambda=\Lambda_{1} \cap \Lambda_{2}$. Let the validity time of the $j-t h$ policy be $\left\{C_{j}^{i}, j=1,2, \cdots\right\}$ with probability $P\left(C_{j}^{i}=\alpha_{\ell}^{i}\right)=p_{\ell}^{i}, \ell=1,2, \cdots, K^{i}$, where they are independent and identically distributed. The premium is $f_{i}\left(C_{j}^{i}\right)$ and $f_{i}(\cdot)$ is a strictly increasing function. $D_{j}^{i}$ is claim time of the $j-t h$ policy and independent and identically distributed function $H_{i}(\cdot) . X_{j}^{i}$ is the $j-t h$ claim size and identically distributed function $F_{i}(\cdot)$. Suppose that $X_{j}^{i}$ have the same distributions $X^{i}$. For any time $t \geq 0$, the surplus process of the insurer can be described as

$$
\begin{aligned}
\left(\begin{array}{l}
R_{1}(t) \\
R_{2}(t)
\end{array}\right) & =\left(\begin{array}{c}
x_{1} e^{\delta t} \\
x_{2} e^{\delta t}
\end{array}\right)+\left(\begin{array}{l}
\sum_{i=1}^{N_{1}(t)} f_{1}\left(C_{i}^{1}\right) e^{\delta\left(t-S_{i}^{1}\right)} \\
\sum_{j=1}^{N_{2}(t)} f_{2}\left(C_{j}^{2}\right) e^{\delta\left(t-S_{j}^{2}\right)}
\end{array}\right) \\
& -\left(\begin{array}{l}
\sum_{i=1}^{N_{1}(t)} X_{i}^{1} e^{\delta\left(t-S_{i}^{1}-D_{i}^{1}\right)} I_{\left\{S_{i}^{1}+D_{i}^{1} \leq t, D_{i}^{1} \leq C_{i}^{1}\right\}} \\
\sum_{j=1}^{N_{2}(t)} X_{j}^{2} e^{\delta\left(t-S_{j}^{2}-D_{j}^{2}\right)} I_{\left\{S_{j}^{2}+D_{j}^{2} \leq t, D_{j}^{2} \leq C_{j}^{2}\right\}}
\end{array}\right) .
\end{aligned}
$$

where $\delta>0$ denotes the constant force of interest. We further assume that $\left\{X_{j}^{1}, j \geq 1\right\},\left\{X_{j}^{2}, j \geq 1\right\}$ and $\left\{\left(N_{1}(t), N_{2}(t)\right)^{\mathrm{T}}, t \geq 0\right\}$ are mutually independent. Define the finite-time ruin probabilities corresponding to risk model (1) as

$$
\begin{aligned}
\psi\left(x_{1}, x_{2} ; t\right) & =P\left(\tau_{\max }\left(x_{1}, x_{2}\right) \leq t\right) \\
& =P\left(\bigcap_{i=1}^{2} R_{i}(s)<0,0 \leq s \leq t\right),
\end{aligned}
$$

where 


$$
\begin{aligned}
& \tau_{\max }\left(x_{1}, x_{2}\right) \\
& =\inf \left\{t: \max \left\{R_{1}(t), R_{2}(t)\right\}<0 \mid R_{i}(0)=x_{i}\right. \\
& , i=1,2\} .
\end{aligned}
$$

In the rest of this paper, Section 2 presents our main results after introducing necessary preliminaries, Section 3 gives some lemmas, and Sections 4 gives the proofs of the main results.

\section{PRELIMINARIES AND MAIN RESULTS}

Definition 1. For a distribution $F$, denote its tail distribution by $\bar{F}=1-F$ and its upper Matuszewska index by

$$
J_{F}^{+}=-\lim _{x \rightarrow \infty} \frac{\log \bar{F}_{*}(x)}{\log x}
$$

where

$$
\bar{F}_{*}(x)=\liminf _{x \rightarrow \infty} \frac{\bar{F}(x y)}{\bar{F}(x)} \text { for } y>0,
$$

which can be found in [8].A distribution $F$ is said to be dominatedly-varying-tailed, denoted by $F \in D$, if for every fixed $y \in(0,1), \bar{F}_{*}(x)<\infty$. Clearly, $F \in D$. A distribution $F$ is said to be long-tailed, denoted by $F \in L$, if for every fixed $y \in R, \bar{F}(x+y) \square \bar{F}(x)$ as $x \rightarrow \infty$, that is,

$$
\lim _{x \rightarrow \infty} \frac{\bar{F}(x+y)}{\bar{F}(x)}=1,
$$

which can be found in [9].

Definition 2. If real valued random variables $X_{i}, i \geq 1$ with distribution functions $F_{i}, i \geq 1$ satisfy for any $i \neq j$

$$
\lim _{\min \left\{x_{i}, x_{j}\right\} \rightarrow \infty} P\left(X_{i}>x_{i} \mid X_{j}>x_{j}\right)=0
$$

Then we say $X_{i}, i \geq 1$ are pairwise strong quasi-asymptotically independent (PSQAI), which can be found in [10].

Theorem 1. Consider the bidimensional risk model (1). Suppose that claim sizes, $\left\{X_{j}^{i}, j \geq 1\right\}$ be PSQAI random variables with common distribution $F_{i} \in D \cap L \quad, i=1,2$. If $N_{1}(t)$ and $N_{2}(t)$ are arbitrarily dependent, then for any fixed $\varepsilon>0$ with $E\left[N_{1}(\varepsilon) N_{2}(\varepsilon)\right]>0$. Then for $t \in \Lambda \bigcap[\varepsilon, T]$, we have $\psi\left(x_{1}, x_{2} ; t\right)$

$$
\begin{aligned}
& \square \int_{0}^{t} \int_{0}^{t} \prod_{i=1}^{2}\left(\sum_{\ell=1}^{K^{i}} p_{\ell}^{i} \int_{0}^{\alpha_{\ell}^{i} \Lambda\left(t-u_{i}\right)} \bar{F}_{i}\left(x_{i} e^{\delta\left(u_{i}+y\right)}\right)\right. \\
& \left.d H_{i}(y)\right) E\left[N_{1}\left(u_{1}\right) N_{2}\left(u_{2}\right)\right] .
\end{aligned}
$$

\section{SOME LEMMAS}

The following lemma is an immediate corollary of Theorems 3.2 and 3.4 of [11].

Lemma 1. Let $\left\{N_{1}(t), t \geq 0\right\}$ and $\left\{N_{2}(t), t \geq 0\right\}$ be two renewal counting processes with their inter-arrival time $\theta_{1}^{1}, \theta_{2}^{1}, \cdots$, and $\theta_{1}^{2}, \theta_{2}^{2}, \cdots$, respectively. For any integer $i \geq 1$, denote by $S_{i}^{1}=\sum_{j=1}^{i} \theta_{j}^{1} \quad$ and $S_{i}^{2}=\sum_{j=1}^{i} \theta_{j}^{2}$ the corresponding arrival times. If $\left\{\left(\theta_{i}^{1}, \theta_{i}^{2}\right) ; i \geq 1\right\}$ is a sequence of i.i.d. random vectors, then it holds for any $u_{1} \geq 0$ and $u_{2} \geq 0$ that

$$
\sum_{i=1}^{\infty} \sum_{j=1}^{\infty} P\left(S_{i}^{1} \leq u_{1}, S_{j}^{2} \leq u_{2}\right)=E\left(N_{1}\left(u_{1}\right) N_{2}\left(u_{2}\right)\right) .
$$

The following lemma comes from Theorem 2.1 of [10]

Lemma 2. Assume that $\left\{X_{j}, 1 \leq j \leq n\right\}$ are $n$ real-valued random variables with functions of distribution $F_{j}, 1 \leq j \leq n$. If $\left\{X_{j}, 1 \leq j \leq n\right\}$ are PSQAI and $F_{j} \in D \bigcap L$ and $\left(c_{1}, \cdots, c_{n}\right) \in[a, b]^{n}$. Then

$$
P\left(\sum_{j=1}^{n} c_{j} X_{j}>x\right) \square \sum_{j=1}^{n} P\left(c_{j} X_{j}>x\right) .
$$

The following lemma comes from proposition 2.2.1 of [9]

Lemma 3. If a distribution $F \in D$, then for any $\beta>J_{F}^{+}$, there exist two positive constant $C$ and $D$ such that for all $x \geq y \geq D$,

$$
\frac{\bar{F}(y)}{\bar{F}(x)} \leq C\left(\frac{x}{y}\right)^{\beta}
$$

Lemma 4. Under the conditions of Theorem 1, it holds that uniformly for $t \in \Lambda \bigcap[\varepsilon, T]$,

$$
\begin{gathered}
P\left(\sum_{i=1}^{N_{1}(t)} X_{i}^{1} e^{-\delta\left(S_{i}^{1}+D_{i}^{1}\right)} I_{\left\{S_{i}^{1}+D_{i}^{1} \leq t, D_{i}^{1} \leq C_{i}^{1}\right\}}>x_{1},\right. \\
\left.\sum_{j=1}^{N_{2}(t)} X_{j}^{2} e^{-\delta\left(S_{j}^{2}+D_{j}^{2}\right)} I_{\left\{S_{j}^{2}+D_{j}^{2} \leq t, D_{j}^{2} \leq C_{j}^{2}\right\}}>x_{2}\right) \\
\square \int_{0}^{t} \int_{0}^{t} \prod_{i=1}^{2}\left(\sum_{\ell=1}^{K^{i}} p_{\ell}^{i} \int_{0}^{\alpha_{\ell}^{i} \Lambda\left(t-u_{i}\right)} \bar{F}_{i}\left(x_{i} e^{\delta\left(u_{i}+y\right)}\right)\right. \\
\left.d H_{i}(y)\right) d E\left[N_{1}\left(u_{1}\right) N_{2}\left(u_{2}\right)\right] .
\end{gathered}
$$

Proof. For arbitrarily fixed positive integer M, we split the left-hand side of (3) into three parts as

$$
\left(\sum_{m=1}^{M} \sum_{n=1}^{M}+\sum_{m=1}^{\infty} \sum_{n=M+1}^{\infty}+\sum_{m=M+1}^{\infty} \sum_{n=1}^{\infty}-\sum_{m=M+1}^{\infty} \sum_{n=M+1}^{\infty}\right)
$$




$$
\begin{gathered}
\times P\left(\sum_{i=1}^{m} X_{i}^{1} e^{-\delta\left(S_{i}^{1}+D_{i}^{1}\right)} I_{\left\{S_{i}^{1}+D_{i}^{1} \leq t, D_{i}^{1} \leq C_{i}^{1}\right\}}>x_{1},\right. \\
\sum_{j=1}^{n} X_{j}^{2} e^{-\delta\left(S_{j}^{2}+D_{j}^{2}\right)} I_{\left\{S_{j}^{2}+D_{j}^{2} \leq t, D_{j}^{2} \leq C_{j}^{2}\right\}}>x_{2}, \\
\left.N_{1}(t)=m, \quad N_{2}(t)=n\right) \\
=\sum_{k=1}^{3} I_{k}\left(x_{1}, x_{2} ; t\right)-I_{4}\left(x_{1}, x_{2} ; t\right) .
\end{gathered}
$$

We first deal with $I_{1}\left(x_{1}, x_{2} ; t\right)$. Write $B_{m}^{1}=\left\{0 \leq z_{1}^{1} \leq \cdots \leq z_{m}^{1} \leq t<z_{m+1}^{1}\right\}$, and $B_{m}^{1}=\{0 \leq$ $\left.\leq \cdots \leq z_{m}^{1} \leq t<z_{m+1}^{1}\right\}$. Since the three sequences

$$
\left\{X_{j}^{i}, j \geq 1\right\}, i=1,2, \text { and }\left\{\left(N_{1}(t), N_{2}(t)^{\mathrm{T}}, t \geq 0\right)\right\}
$$

are mutually independent, and using Lemma 2, we have that uniformly for $t \in \Lambda \bigcap[\varepsilon, T]$

$$
\begin{aligned}
& I_{1}\left(x_{1}, x_{2} ; t\right) \\
& =\sum_{m=1}^{M} \sum_{n=1}^{M} P\left(\sum_{i=1}^{m} X_{i}^{1} e^{-\delta\left(S_{i}^{1}+D_{i}^{1}\right)} I_{\left\{S_{i}^{1}+D_{i}^{1} \leq t, D_{i}^{1} \leq C_{i}^{1}\right\}}>x_{1},\right. \\
& \sum_{j=1}^{n} X_{j}^{2} e^{-\delta\left(S_{j}^{2}+D_{j}^{2}\right)} I_{\left\{S_{j}^{2}+D_{j}^{2} \leq t, D_{j}^{2} \leq C_{j}^{2}\right\}}>x_{2} \text {, } \\
& \left.N_{1}(t)=m, \quad N_{2}(t)=n\right) \\
& =\sum_{m=1}^{M} \sum_{n=1}^{M} \int \cdots \int_{B_{m}^{1} ; B_{n}^{2}} \\
& P\left(\sum_{i=1}^{m} X_{i}^{1} e^{-\delta\left(S_{i}^{1}+D_{i}^{1}\right)} I_{\left\{S_{i}^{1}+D_{i}^{1} \leq t, D_{i}^{1} \leq C_{i}^{1}\right\}}>x_{1},\right. \\
& \left.\sum_{j=1}^{n} X_{j}^{2} e^{-\delta\left(S_{j}^{2}+D_{j}^{2}\right)} I_{\left\{S_{j}^{2}+D_{j}^{2} \leq t, D_{j}^{2} \leq C_{j}^{2}\right\}}>x_{2}\right) \\
& \times P\left(S_{1}^{1} \in d z_{1}^{1}, \cdots, S_{m+1}^{1} \in d z_{m+1}^{1},\right. \\
& \left.S_{1}^{2} \in d z_{1}^{2}, \cdots, S_{n+1}^{2} \in d z_{n+1}^{2}\right) \\
& \square \sum_{m=1}^{M} \sum_{n=1}^{M} \sum_{i=1}^{m} \sum_{j=1}^{n} \int \cdots \int_{B_{m}^{1} ; B_{n}^{2}} \\
& P\left(X_{i}^{1} e^{-\delta\left(S_{i}^{1}+D_{i}^{1}\right)} I_{\left\{S_{i}^{1}+D_{i}^{1} \leq t, D_{i}^{1} \leq C_{i}^{1}\right\}}>x_{1}\right) \\
& P\left(X_{j}^{2} e^{-\delta\left(S_{j}^{2}+D_{j}^{2}\right)} I_{\left\{S_{j}^{2}+D_{j}^{2} \leq t, D_{j}^{2} \leq C_{j}^{2}\right\}}>x_{2}\right) \\
& \times P\left(S_{1}^{1} \in d z_{1}^{1}, \cdots, S_{m+1}^{1} \in d z_{m+1}^{1},\right. \\
& \left.S_{1}^{2} \in d z_{1}^{2}, \cdots, S_{n+1}^{2} \in d z_{n+1}^{2}\right) \\
& =\sum_{m=1}^{M} \sum_{n=1}^{M} \sum_{i=1}^{m} \sum_{j=1}^{n} P\left(X_{i}^{1} e^{-\delta\left(S_{i}^{1}+D_{i}^{1}\right)} I_{\left\{S_{i}^{1}+D_{i}^{1} \leq t, D_{i}^{1} \leq C_{i}^{1}\right\}}>x_{1}\right. \text {, } \\
& X_{j}^{2} e^{-\delta\left(S_{j}^{2}+D_{j}^{2}\right)} I_{\left\{S_{j}^{2}+D_{j}^{2} \leq t, D_{j}^{2} \leq C_{j}^{2}\right\}}>x_{2}, N_{1}(t)=m, \\
& \left.N_{2}(t)=n\right) \\
& \square\left(\sum_{m=1}^{\infty} \sum_{n=1}^{\infty}-\left(\sum_{m=1}^{\infty} \sum_{n=M+1}^{\infty}+\sum_{m=M+1}^{\infty} \sum_{n=1}^{M}\right)\right)
\end{aligned}
$$

$$
\begin{aligned}
& \sum_{i=1}^{m} \sum_{j=1}^{n} P\left(X_{i}^{1} e^{-\delta\left(S_{i}^{1}+D_{i}^{1}\right)} I_{\left\{S_{i}^{1}+D_{i}^{1} \leq t, D_{i}^{1} \leq C_{i}^{1}\right\}}>x_{1},\right. \\
& X_{j}^{2} e^{-\delta\left(S_{j}^{2}+D_{j}^{2}\right)} I_{\left\{S_{j}^{2}+D_{j}^{2} \leq t, D_{j}^{2} \leq C_{j}^{2}\right\}}>x_{2}, N_{1}(t)=m, \\
& \left.N_{2}(t)=n\right) \\
& =J_{1}\left(x_{1}, x_{2} ; t\right)-J_{2}\left(x_{1}, x_{2} ; t\right)
\end{aligned}
$$

For $J_{1}\left(x_{1}, x_{2} ; t\right)$, uniformly for all $t \in \Lambda \bigcap[\varepsilon, T]$

$$
\begin{aligned}
& J_{1}\left(x_{1}, x_{2} ; t\right) \\
& =\sum_{j=1}^{\infty} \sum_{i=1}^{\infty} P\left(X_{i}^{1} e^{-\delta\left(S_{i}^{1}+D_{i}^{1}\right)} I_{\left\{S_{i}^{1}+D_{i}^{1} \leq t, D_{i}^{1} \leq C_{i}^{1}\right\}}>x_{1},\right. \\
& X_{j}^{2} e^{-\delta\left(S_{j}^{2}+D_{j}^{2}\right)} I_{\left\{S_{j}^{2}+D_{j}^{2} \leq t, D_{j}^{2} \leq C_{j}^{2}\right\}}>x_{2}, S_{i}^{1} \leq t, \\
& \left.S_{j}^{2} \leq t\right) \\
& =\sum_{j=1}^{\infty} \sum_{i=1}^{\infty} \int_{0}^{t} \int_{0}^{t} \prod_{i=1}^{2}\left(\sum_{\ell=1}^{K^{i}} p_{\ell}^{i} \int_{0}^{\alpha_{\ell}^{i} \Lambda\left(t-u_{i}\right)} \bar{F}_{i}\left(x_{i} e^{\delta\left(u_{i}+y\right)}\right)\right. \\
& \left.\quad d H_{i}(y)\right) P\left(S_{i}^{1} \in d u_{1}, S_{j}^{2} \in d u_{2}\right) \\
& =\int_{0}^{t} \int_{0}^{t} \prod_{i=1}^{2}\left(\sum_{\ell=1}^{K^{i}} p_{\ell}^{i} \int_{0}^{\alpha_{\ell}^{i} \Lambda\left(t-u_{i}\right)} \bar{F}_{i}\left(x_{i} e^{\delta\left(u_{i}+y\right)}\right)\right. \\
& \\
& \left.d H_{i}(y)\right) d E\left[N_{1}\left(u_{1}\right) N_{2}\left(u_{2}\right)\right] .
\end{aligned}
$$

As for $J_{2}\left(x_{1}, x_{2} ; t\right)$, we have that for all $t \in \Lambda \bigcap[\varepsilon, T]$,

$$
\begin{gathered}
J_{2}\left(x_{1}, x_{2} ; t\right) \leq\left(\sum_{m=1}^{\infty} \sum_{n=M+1}^{\infty}+\sum_{m=M+1}^{\infty} \sum_{n=1}^{\infty}\right) \\
\sum_{i=1}^{m} \sum_{j=1}^{n} P\left(X_{i}^{1}>x_{1}, X_{j}^{2}>x_{2},\right. \\
\left.N_{1}(t)=m, \quad N_{2}(t)=n\right) \\
=K_{1}\left(x_{1}, x_{2} ; t\right)+K_{2}\left(x_{1}, x_{2} ; t\right) .
\end{gathered}
$$

It holds that for all $t \in \Lambda \bigcap[\varepsilon, T]$,

$$
\begin{aligned}
& K_{1}\left(x_{1}, x_{2} ; t\right) \\
& \leq \sum_{m=1}^{\infty} \sum_{n=M+1}^{\infty} \sum_{i=1}^{m} \sum_{j=1}^{n} P\left(X_{i}^{1}>x_{1}, X_{j}^{2}>x_{2}, N_{1}(t)=m,\right. \\
& \left.\quad N_{2}(t)=n\right) \\
& =P\left(X_{i}^{1}>x_{1}, X_{j}^{2}>x_{2}\right) \sum_{m=1}^{\infty} \sum_{n=M+1}^{\infty} m n P\left(N_{1}(t)=m,\right. \\
& \left.\quad N_{2}(t)=n\right) . \\
& =\bar{F}\left(x_{1}\right) \bar{F}\left(x_{2}\right) E\left(N_{1}(t) N_{2}(t) I_{N_{2}(T)>M}\right) .
\end{aligned}
$$

We obtain from Hölder's inequality that

$$
\sup _{t \in \Lambda[\varepsilon, T], M \rightarrow \infty} \frac{E\left(N_{1}(t) N_{2}(t) I_{N_{2}(T)>M}\right)}{E\left(N_{1}(t) N_{2}(t)\right)}
$$




$$
\leq \frac{\left(E\left[\left(N_{1}(T)\right)\right]^{2}\right)^{\frac{1}{2}}\left(E\left[\left(N_{2}(T)\right)^{2} I_{\left\{N_{2}(T)>M\right\}}\right]\right)^{\frac{1}{2}}}{E\left(N_{1}(\varepsilon) N_{2}(\varepsilon)\right)}=0 .
$$

Then, by $F_{i} \in D, i=1,2$, we have

$$
\begin{aligned}
& \lim _{M \rightarrow \infty} \lim _{\min \left\{x_{1}, x_{2}\right\} \rightarrow \infty} \sup _{t \in \Lambda \cap(0, T]} \\
& \int_{0}^{t} \int_{0}^{t} \prod_{i=1}^{2}\left(\sum_{\ell=1}^{K^{i}} p_{\ell}^{i} \int_{0}^{\alpha_{\ell}^{i} \Lambda\left(t-u_{i}\right)} \bar{F}_{i}\left(x_{i} e^{\delta\left(u_{i}+y\right)}\right)\right. \\
& \times \frac{1}{\left.d H_{i}(y)\right) d E\left[N_{1}\left(u_{1}\right) N_{2}\left(u_{2}\right)\right]} \\
& \leq \lim _{\min \left\{x_{1}, x_{2}\right\} \rightarrow \infty} \frac{\bar{F}\left(x_{1}\right) \bar{F}\left(x_{2}\right)}{\bar{F}_{1}\left(x_{1} e^{\delta T}\right) \bar{F}_{2}\left(x_{2} e^{\delta T}\right)} \\
& \quad \times \frac{1}{\prod_{i=1}^{2} \sum_{\ell=1}^{K^{i}} p_{\ell}^{i} H_{i}\left(\alpha_{\ell}^{i} \wedge\left(t-u_{i}\right)\right)} \\
& \times \lim _{M \rightarrow \infty} \sup _{t \in \Lambda(0, T]} \frac{E\left(N_{1}(t) N_{2}(t) I_{N_{2}(T)>M}\right)}{E\left(N_{1}(t) N_{2}(t)\right)}=0 .
\end{aligned}
$$

$K_{2}\left(x_{1}, x_{2} ; t\right)$ can be dealt with in the same way. Thus, we obtain

$$
\begin{aligned}
& \lim _{M \rightarrow \infty} \lim _{\min \left\{x_{1}, x_{2}\right\} \rightarrow \infty} \sup _{t \in \Lambda \cap(0, T]} \\
& \frac{J_{2}\left(x_{1}, x_{2} ; t\right)}{\int_{0}^{t} \int_{0}^{t} \prod_{i=1}^{2}\left(\sum_{\ell=1}^{K^{i}} p_{\ell}^{i} \int_{0}^{\alpha_{\ell}^{i} \Lambda\left(t-u_{i}\right)} \overline{F_{i}}\left(x_{i} e^{\delta\left(u_{i}+y\right)}\right)\right.} \\
& \times \frac{1}{\left.d H_{i}(y)\right) E\left(N_{1}(t) N_{2}(t)\right)}=0 .
\end{aligned}
$$

Substituting (6) and (8) into (5) leads to $I_{1}\left(x_{1}, x_{2} ; t\right)$

$$
\begin{gathered}
\square \int_{0}^{t} \int_{0}^{t} \prod_{i=1}^{2}\left(\sum_{\ell=1}^{K^{i}} p_{\ell}^{i} \int_{0}^{\alpha_{\ell}^{i} \Lambda\left(t-u_{i}\right)} \bar{F}_{i}\left(x_{i} e^{\delta\left(u_{i}+y\right)}\right)\right. \\
\left.d H_{i}(y)\right) d E\left[N_{1}\left(u_{1}\right) N_{2}\left(u_{2}\right)\right] .
\end{gathered}
$$

We next estimate $I_{2}\left(x_{1}, x_{2} ; t\right)$. Choose some $\beta>\max \left\{J_{F_{1}}^{+}, J_{F_{2}}^{+}\right\}$. According to Lemma 3 , uniformly for all $t \in \Lambda \bigcap[\varepsilon, T]$,

$$
\begin{aligned}
& I_{2}\left(x_{1}, x_{2} ; t\right) \\
& \leq \sum_{m=1}^{\infty} \sum_{n=M+1}^{\infty} m n P\left(X^{1}>x_{1} / m\right) P\left(X^{2}>x_{2} / n\right) \\
& \quad \times P\left(N_{1}(t)=m, \quad N_{2}(t)=n\right) \\
& \leq C_{1} C_{2} P\left(X^{1}>x_{1}\right) P\left(X^{2}>x_{2}\right) \sum_{m=1}^{\infty} \sum_{n=M+1}^{\infty} m^{\beta+1} n^{\beta+1} \\
& \quad \times P\left(N_{1}(t)=m, \quad N_{2}(t)=n\right)
\end{aligned}
$$

$$
\begin{aligned}
\leq & C P\left(X^{1}>x_{1}\right) P\left(X^{2}>x_{2}\right) \\
& \times E\left(N_{1}(t)^{\beta+1} N_{2}(t)^{\beta+1} I_{N_{2}(T)>M}\right),
\end{aligned}
$$

where $C$ is some positive constant. Similarly to (8) we obtain

$$
\begin{aligned}
& \lim _{M \rightarrow \infty} \lim _{\min \left\{x_{1}, x_{2}\right\} \rightarrow \infty} \sup _{t \in \Lambda \cap(0, T]} \\
& \frac{I_{2}\left(x_{1}, x_{2} ; t\right)}{\int_{0}^{t} \int_{0}^{t} \prod_{i=1}^{2}\left(\sum_{\ell=1}^{K^{i}} p_{\ell}^{i} \int_{0}^{\alpha_{\ell}^{i} \Lambda\left(t-u_{i}\right)} \bar{F}_{i}\left(x_{i} e^{\delta\left(u_{i}+y\right)}\right)\right.} \\
& \times \frac{1}{\left.d H_{i}(y)\right) d E\left[N_{1}\left(u_{1}\right) N_{2}\left(u_{2}\right)\right]}=0 .
\end{aligned}
$$

In the same manner, we can prove

$$
\begin{aligned}
& \lim _{M \rightarrow \infty} \lim _{\min \left\{x_{1}, x_{2}\right\} \rightarrow \infty} \sup _{t \in \Lambda \cap(0, T]} \\
& \frac{I_{3}\left(x_{1}, x_{2} ; t\right)}{\int_{0}^{t} \int_{0}^{t} \prod_{i=1}^{2}\left(\sum_{\ell=1}^{K^{i}} p_{\ell}^{i} \int_{0}^{\alpha_{\ell}^{i} \Lambda\left(t-u_{i}\right)} \bar{F}_{i}\left(x_{i} e^{\delta\left(u_{i}+y\right)}\right)\right.} \\
& \times \frac{1}{\left.d H_{i}(y)\right) d E\left[N_{1}\left(u_{1}\right) N_{2}\left(u_{2}\right)\right]} \\
& \leq \lim _{M \rightarrow \infty} \lim _{\min \left\{x_{1}, x_{2}\right\} \rightarrow \infty} \sup _{t \in \Lambda \cap(0, T]}
\end{aligned}
$$

$$
\begin{aligned}
& \frac{I_{4}\left(x_{1}, x_{2} ; t\right)}{\int_{0}^{t} \int_{0}^{t} \prod_{i=1}^{2}\left(\sum_{\ell=1}^{K^{i}} p_{\ell}^{i} \int_{0}^{\alpha_{\ell}^{i} \Lambda\left(t-u_{i}\right)} \overline{F_{i}}\left(x_{i} e^{\delta\left(u_{i}+y\right)}\right)\right.} \\
& \times \frac{1}{\left.d H_{i}(y)\right) d E\left[N_{1}\left(u_{1}\right) N_{2}\left(u_{2}\right)\right]} .
\end{aligned}
$$

Substituting (9), (10) and (11) into (4) the desired relation (3) holds uniformly for all $t \in \Lambda \bigcap[\varepsilon, T]$.

\section{PROOF OF MAIN RESULTS.}

Proof of Theorem 1. We first deal with the asymptotic upper bound of $\psi\left(x_{1}, x_{2} ; t\right)$. By Lemma 4 , we have that uniformly for $t \in \Lambda \bigcap[\varepsilon, T]$,

$$
\begin{aligned}
\psi\left(x_{1}, x_{2} ; t\right) \leq & P\left(\sum_{i=1}^{N_{1}(t)} X_{i}^{1} e^{-\delta\left(S_{i}^{1}+D_{i}^{1}\right)} I_{\left\{S_{i}^{1}+D_{i}^{1} \leq t, D_{i}^{1} \leq C_{i}^{1}\right\}}>x_{1},\right. \\
& \left.\sum_{j=1}^{N_{2}(t)} X_{j}^{2} e^{-\delta\left(S_{j}^{2}+D_{j}^{2}\right)} I_{\left\{S_{j}^{2}+D_{j}^{2} \leq t, D_{j}^{2} \leq C_{j}^{2}\right\}}>x_{2}\right) \\
\square & \int_{0}^{t} \int_{0}^{t} \prod_{i=1}^{2}\left(\sum_{\ell=1}^{K^{i}} p_{\ell}^{i} \int_{0}^{\alpha_{\ell}^{i} \Lambda\left(t-u_{i}\right)} \bar{F}_{i}\left(x_{i} e^{\delta\left(u_{i}+y\right)}\right)\right. \\
& \left.d H_{i}(y)\right) d E\left[N_{1}\left(u_{1}\right) N_{2}\left(u_{2}\right)\right] .
\end{aligned}
$$

Then we discuss the asymptotic lower bound of $\psi\left(x_{1}, x_{2} ; t\right)$. For simplicity, write

$$
Z_{k}(t)=\sum_{i=1}^{N_{k}(t)} f_{k}\left(C_{i}^{k}\right) e^{-\delta S_{i}^{k}}, \quad k=1,2 .
$$

For sufficiently large $N>0$, by Lemma 4, 
$F_{k} \in L, k=1,2$, we obtain that uniformly for $t \in \Lambda \bigcap[\varepsilon, T]$,

$$
\begin{aligned}
& \psi\left(x_{1}, x_{2} ; t\right) \\
& \square \int_{0}^{\infty} \int_{0}^{\infty} P\left(\sum_{i=1}^{N_{1}(t)} X_{i}^{1} e^{-\delta\left(S_{i}^{1}+D_{i}^{1}\right)} I_{\left\{S_{i}^{1}+D_{i}^{1} \leq t, D_{i}^{1} \leq C_{i}^{1}\right\}}\right. \\
& >x_{1}+z_{1} \text {, } \\
& \left.\sum_{j=1}^{N_{2}(t)} X_{j}^{2} e^{-\delta\left(S_{j}^{2}+D_{j}^{2}\right)} I_{\left\{S_{j}^{2}+D_{j}^{2} \leq t, D_{j}^{2} \leq C_{j}^{2}\right\}}>x_{2}+z_{2}\right) \\
& P\left(Z_{1}(t) \in d z_{1}, Z_{2}(t) \in d z_{2}\right) \\
& \square \int_{0}^{\infty} \int_{0}^{\infty} \int_{0}^{t} \int_{0}^{t} \prod_{i=1}^{2}\left(\sum_{\ell=1}^{K^{i}} p_{\ell}^{i} \int_{0}^{\alpha_{\ell}^{i} \Lambda\left(t-u_{i}\right)} \bar{F}_{i}\left(x_{i} e^{\delta\left(u_{i}+y\right)}\right)\right. \\
& \left.d H_{i}(y)\right) d E\left[N_{1}\left(u_{1}\right) N_{2}\left(u_{2}\right)\right] \\
& \times P\left(Z_{1}(t) \in d z_{1}, Z_{2}(t) \in d z_{2}\right) \\
& \geq \int_{0}^{N} \int_{0}^{N} \int_{0}^{t} \int_{0}^{t} \prod_{i=1}^{2}\left(\sum_{\ell=1}^{K^{i}} p_{\ell}^{i} \int_{0}^{\alpha_{\ell}^{i} \Lambda\left(t-u_{i}\right)} \bar{F}_{i}\left(x_{i} e^{\delta\left(u_{i}+y\right)}\right)\right. \\
& \left.d H_{i}(y)\right) d E\left[N_{1}\left(u_{1}\right) N_{2}\left(u_{2}\right)\right] \\
& \times P\left(Z_{1}(t) \in d z_{1}, Z_{2}(t) \in d z_{2}\right) \\
& \square P\left(Z_{1}(t) \leq N, Z_{2}(t) \leq N\right) \\
& \int_{0}^{t} \int_{0}^{t} \prod_{i=1}^{2}\left(\sum_{\ell=1}^{K^{i}} p_{\ell}^{i} \int_{0}^{\alpha_{\ell}^{i} \Lambda\left(t-u_{i}\right)} \bar{F}_{i}\left(x_{i} e^{\delta\left(u_{i}+y\right)}\right) d H_{i}(y)\right) \\
& d E\left[N_{1}\left(u_{1}\right) N_{2}\left(u_{2}\right)\right] \\
& \square \int_{0}^{t} \int_{0}^{t} \prod_{i=1}^{2}\left(\sum_{\ell=1}^{K^{i}} p_{\ell}^{i} \int_{0}^{\alpha_{\ell}^{i} \Lambda\left(t-u_{i}\right)} \bar{F}_{i}\left(x_{i} e^{\delta\left(u_{i}+y\right)}\right)\right. \\
& \left.d H_{i}(y)\right) d E\left[N_{1}\left(u_{1}\right) N_{2}\left(u_{2}\right)\right] \text {. }
\end{aligned}
$$

A combination of (12) and (13) shows that (2) holds uniformly for all $t \leq T$

\section{REFERENCES}

[1] Z. Li, J. Zhu, F. Chen, Study of a risk model based on the entrance process[J]. Statistics and Probability Letters. 2005, 72(1):1-10.

[2] Z. Li, X. Kong, A new risk model based on policy entrance process and its weak convergence properties [J]. Applied Stochastic Models in Business and Industry. 2007, 23(3): 235-246.

[3] H. Xiao, J. Tang, Asymptotic ruin probabilities of an entrance processes based risk model with interest force and regularly varying claims $[\mathrm{J}]$. Chinese Journal of Engineering Mathematics. 2009, 26(6): 1126-1132.

[4] H. Xiao, Z. LI, W. Liu, The finite time ruin probability of a new risk model based on entrance process[J]. Communications in Statistics-Theory and Methods. 2013, 42(2): 336-345.

[5] F. Tang, J. Bai, Precise large deviations for aggregate loss process in a multi-risk model[J]. J. Korean Math. Soc. 2015, 52(3): 447-467.

[6] H. Xiao, L. Xie, Asymptotic ruin probability of a bidimensional risk model based on entrance processes with constant interest rate $[\mathrm{J}]$. Communications in Statistics-Theory and Methods. 2018, 47(22): 5396-5417.

[7] H. Yang, J. Li, Asymptotic ruin probabilities for a bidimensional renewal risk model, Stochastics. 2017, 89(5): 687-708.

[8] Y. Yang, Y. Wang, Asymptotics for ruin probability of some negatively dependent risk models with a constant interest rate and dominatedly-varying-tailed claims[J]. Statist. Probab. Lett. 2010,80(3): 143-154.

[9] N. Bingham,C. Goldie, J. Teugels, Regular variation[M] Cambridge: Cambridge University Press, 1987.

[10] J. Li, On pairwise quasi-asymptotically independent random variables and their applications[J]. Statist. Probab. Lett.
2013,83(9):2081-2087.

[11] J. Hunter, Renewal theory in two dimensions: Basic results[J]. Advances in Appl. Probability. 1974, 6(2): 376-391.

Zhankui Wang, School of Mathematics and Statistics, Northwest Norma University, Lanzhou, China, Mobile 86- 18894575336. 